\title{
ON A MINIMALITY PROPERTY OF COMPLEXES
}

\author{
JOSEPH ZAKS ${ }^{1}$
}

I. Introduction and results. It is well known that any finite $n$-complex ( $n$-dimensional complex) is geometrically embeddable in $E^{2 n+1}$, and that there are $n$-complexes which are not embeddable, even topologically, in $E^{2 n}$; the last result is due to A. Flores [1] (see also E. R. van Kampen [3]; for additional proofs see R. H. Rosen [5] and W. T. Wu [6]).

Extending the previous results, B. Grünbaum [2] used a similar proof to establish:

ThEOREM 1. Let $n, p, n_{1}, \cdots, n_{p}$ be positive integers, such that $n_{1}+\cdots+n_{p}=n+1$, then the complex

$$
C_{2 n_{1}+1}^{n_{1}-1} \vee \cdots \vee C_{2 n_{p}+1}^{n_{p}-1}
$$

is an n-complex, which is not embeddable in $E^{2 n}$.

Here $C_{a}^{p}$ denotes the complete $p$-complex with $q$ vertices, and $K \vee L$ is the join complex of the complexes $K$ and $L$.

We shall call an $n$-complex of the form (i) nice.

A geometrical embedding of a complex $K$ in $E^{n}$ is an embedding which is linear, with respect to the barycentric coordinates, on each simplex of $K$. A piecewise linear (p.w.l.) embedding is to be understood in the usual way. All complexes are assumed finite.

The purpose of this paper is to prove the following minimality property of all the nice $n$-complexes:

TheOREM 2. Each proper subcomplex of a nice n-complex is p.w.l. embeddable in $E^{2 n}$.

A partial improvement is the following:

TheOREM 3. Each proper subcomplex of a nice n-complex is geometrically embeddable in $E^{2 n}$, provided

(a) $n_{i}=1$ for all, except possibly one of the $i-s$, or

(b) $n_{1}=n+1$.

For arbitrary complexes we have the following two results:

Theorem 4. If all the proper subcomplexes of a complex $K$ are geo1968.

Presented to the Society, April 12, 1968; received by the editors February 26,

1 Research supported in part by the National Science Foundation Grant GP-7035. 
metrically embeddable in $E^{k}$, then each proper subcomplex of $K \bigvee C_{3}^{0}$ is geometrically embeddable in $E^{k+2}$.

THEOREM 5. If all the proper subcomplexes of the complexes $K$ and $L$ are p.w.l. embeddable in $E^{k}$ and $E^{d}$, respectively, then each proper subcomplex of $K \bigvee L$ is p.w.l. embeddable in $E^{k+d+2}$.

Notice that, in the last two theorems, the dimensions $k$ and $d$ are in no relation with the dimensions of the complexes $K$ and $L$, respectively. Though some necessary and sufficient conditions for p.w.l. embeddability of an $n$-complex in $E^{2 n}$ are known for $n>2$, (see W. T. $\mathrm{Wu}$ [6], Theorems 1 and 2 of Chapter VI, §4), we doubt that they can be simply applied to our Theorem 2 . Such a condition, in the case $n=1$, is very well known, due to C. Kuratowski [4].

Theorem 3, part (b) was proved by B. Grünbaum [2], and we include his proof, for completeness.

The author would like to thank Professor Branko Grünbaum for posing the above mentioned problems, as well as for his patience to hear these results and also the referee for his useful remarks.

II. Notations and proofs. A simplex $\sigma$ of a complex $K, \sigma \in K$, is called maximal if $\sigma$ is not a proper face of any simplex of $K$. A proper subcomplex $L$ of a complex $K$ is called maximal if $L$ is a proper subcomplex of no other proper subcomplex of $K$. If $\sigma$ is a simplex of a complex $K, \mathrm{Bd} \sigma$ will denote that subcomplex of $K$ which contains precisely all the proper faces of $\sigma ; K-\sigma$ is the collection of all the simplexes of $K$, except $\sigma$, and it is a complex if and only if $\sigma$ is maximal in $K$.

If $L$ is a subcomplex of a complex $K$, and $K$ is (p.w.l., geometrically) embedded in $E^{k},|K|$ will denote its realization in $E^{k}$, and $|L|$ will be the subset of $|K|$ in the usual way.

Although the join $\bigvee$ is an operation defined for abstract complexes, there is no confusion in applying it to geometrical complexes, provided they are situated properly with respect to each other, for example-if they are contained in skew affine flats. Having this in mind, the proofs can be easily described.

For $n<m$, we always use the notation

$$
E^{m} \supset E^{n}=\left\{\left(x_{1}, \cdots, x_{n}, 0, \cdots, 0\right) \in E^{m}\right\} .
$$

1. Proof of Theorem 5. It is obviously enough to prove that each maximal proper subcomplex $M$ of $K \bigvee L$ is embeddable in $E^{k+d+2}$. Let $M$ be such a complex, then it is clear that $M=K \bigvee L-\sigma \bigvee \eta$, where $\sigma$ is a maximal simplex of $K$, and $\eta$ is a maximal simplex of $L$. 
Using the assumptions on $K$ and $L$, it follows that

$$
|K-\sigma| \subset E^{k} \subset E^{k+d+2},
$$

and

$$
|L-\eta| \subset E_{0}{ }^{d}=\left(0, \cdots, 0, x_{k+1}, \cdots, x_{k+d}, 1,0\right) \in E^{k+d+2}
$$

and since $E^{k}$ and $E_{0}^{d}$ are skew, it follows that

$$
(|K-\sigma| \vee|L-\eta|) \subset E^{k+d+1} \subset E^{k+d+2} \text {. }
$$

Let $P_{i}=\left(0, \cdots, 0,(-1)^{i}\right) \in E^{k+d+2}$, for $i=1,2$; since $P_{i}$ is skew with respect to $E^{k+d+1}$, for $i=1,2$, the following joins are well defined:

$$
|K-\sigma| \vee|L-\eta| \vee P_{i}, \quad i=1,2
$$

Therefore, in particular,

$[|K-\sigma| \vee|L-\eta|]$

$$
\cup\left[|\operatorname{Bd} \sigma| \vee|L-\eta| \vee P_{1}\right] \cup\left[|K-\sigma| \vee|\operatorname{Bd} \eta| \vee P_{2}\right]
$$

is well defined and represents $|M|$ under a p.w.l. embedding, where $|\operatorname{Bd} \sigma| \vee P_{1}$ is homeomorphic to $|\sigma|$, and similarly $|\operatorname{Bd} \eta| \vee P_{2}$ to $|\eta|$.

2. Proof of Theorem 4. Using the beginning of the last proof, let $M$ be a maximal proper subcomplex of $K \bigvee C_{3}^{\mathbf{0}}$, then $M$ $=K \bigvee C_{3}^{0}-\sigma \bigvee A_{1}$ where $\sigma$ is a maximal simplex of $K$, and $A_{1}$ is a vertex of $C_{3}^{0}$.

Using the assumption on $K$, it follows that

$$
|K-\sigma| \subset E^{k} \subset E^{k+2}
$$

where the realization is taken to be geometric.

Let $P_{1}=(0, \cdots, 0,1,0) \in E^{k+2}$; then

$$
|K-\sigma| \vee P_{1} \subset E_{+}^{k+1}=\left\{\left(x_{1}, \cdots, x_{k+1}, 0\right) \in E^{k+2} \mid x_{k+1} \geqq 0\right\}
$$

where $|K-\sigma| \vee P_{1}$ is a geometric realization of $(K-\sigma) \vee P_{1}$ in $E_{+}^{k+1}$.

Let $u_{0}, \cdots, u_{r}$ be the vertices of $\sigma$ in $|K-\sigma| \vee P_{1}$ in $E_{+}^{k+1}$, and let $u_{0}=\left(y_{1}, \cdots, y_{k}, 0,0\right)$, then we define, for some $\epsilon>0, u_{0}^{\prime}$ $=\left(y_{1}, \cdots, y_{k},-\epsilon, 0\right) \in E^{k+2}$.

By pushing $u_{0}$ to $u_{0}^{\prime}$, we get another geometric realization, say $\left(|K-\sigma| \vee P_{1}\right)^{\prime}$ of $(K-\sigma) \vee P_{1}$ in $E^{k+1} ;\left(|K-\sigma| \vee P_{1}\right)^{\prime}$ has the property that if $\eta$ is a simplex of it, which contains a point having a negative $(k+1)$ st coordinate, then $u_{0}^{\prime} \in \eta$.

Therefore we can add to $\left(|K-\sigma| \vee P_{1}\right)^{\prime}$ the convex hull of $\left\{u_{0}^{\prime}, u_{1}, \cdots, u_{r}\right\}$, without interfering with the interior of other simplexes, this means that the complex $\left[(K-\sigma) \vee P_{1}\right] \cup \sigma$ is geometri- 
cally realizable in $E^{k+1}$. Let $P_{i}=\left(0, \cdots, 0,(-1)^{i}\right) \in E^{k+2}$, for $i=2,3$, since $K \subset\left[(K-\sigma) \bigvee P_{1}\right] \cup \sigma$, it follows that

$$
\left(\left|\left[(K-\sigma) \vee P_{1}\right] \cup \sigma\right|\right) \cup\left(|K| \vee P_{2}\right) \cup\left(|K| \vee P_{3}\right) \subset E^{k+2}
$$

represents a geometric realization of $M$ in $E^{k+2}$, where the $P_{i}-s$ are the vertices of $\left|C_{3}^{0}\right|$.

3. Proof of Theorem 3 (b). (This proof is taken from [2].) To show that each proper subcomplex of $C_{2 n+3}^{n}$, for positive $n$, is geometrically realizable in $E^{2 n}$, it is obviously enough to prove the assertion for each maximal proper subcomplex $M$ of $C_{2 n+3}^{n}$. In this case $M$ $=C_{2 n+3}^{n}-\sigma$, where $\sigma$ is an $n$-simplex.

Let $\left|T_{1}\right|$ and $\left|T_{2}\right|$ be two geometric $n$-simplexes in $E^{2 n}$, such that $\left|T_{1}\right| \cap\left|T_{2}\right|$ is a single point, relatively interior to both $\left|T_{1}\right|$ and $\left|T_{2}\right|$.

Let $\left|L_{n}\right|=$ convex hull $\left|T_{1}\right| \cup\left|T_{2}\right| .\left|L_{n}\right|$ is a $2 n$-dimensional convex polytope in $E^{2 n}$, with $2 n+2$ vertices. The $n$-skeleton $B_{n}$ of the boundary simplicial complex of $\left|L_{n}\right|$ contains all the $n$-simplexes determined by the $2 n+2$ vertices of $L_{n}$, except $T_{1}$ and $T_{2}$, as well as all the $(n-1)$-simplexes determined by these $2 n+2$ vertices.

Taking, if necessary, a suitable projective image of $\left|L_{n}\right|$, we may assume, without loss of generality, that there exists a point $\omega \in E^{2 n}$, that is beyond all but one $(2 n-1)$-dimensional faces of $\left|L_{n}\right|$, (e.g.all the boundary of $\left|L_{n}\right|$, except the relative interior of one $(2 n-1)$ dimensional face, "can be seen" from $\omega)$. Therefore $\omega \vee(\mid(n-1)$ skeleton of $\left.B_{n} \mid\right)$ intersects $\left|B_{n}\right|$ only in its $(n-1)$-skeleton, and

$$
\left|T_{1}\right| \cup\left|B_{n}\right| \cup\left[\omega \vee \mid(n-1) \text {-skeleton of } B_{n} \mid\right]
$$

represents a geometrical simplicial complex in $E^{2 n}$, isomorphic to $|M|$, where the vertices of $|\sigma|$ corresponds to the vertices of $\left|T_{2}\right|$.

For the case $n=1$, see Figure 1.
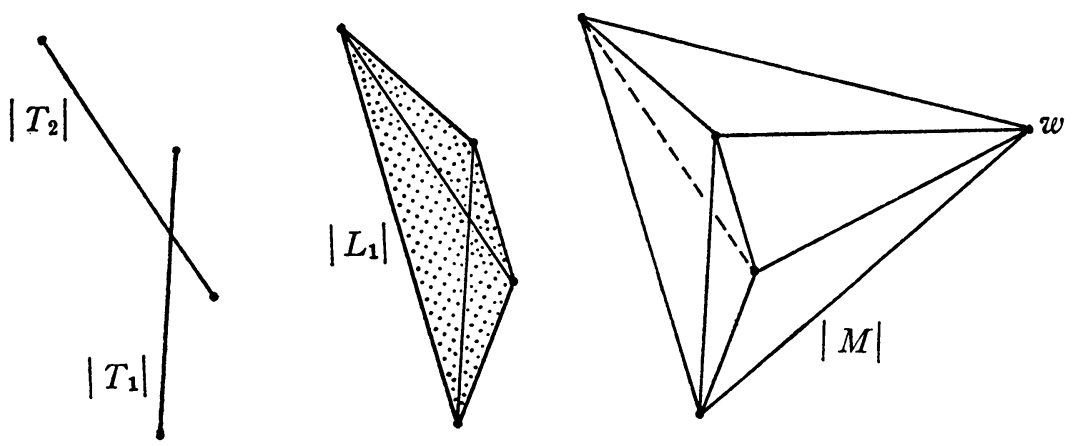

FIGURE 1 
4. Proof of Theorem 3 (a). Case I. All $n_{i}=1$. Then the complex is $C_{3}^{0} \vee \cdots \vee C_{3}^{0}$. From $n \geqq 1$ it follows that $p \geqq 2$. The proof is by induction on $p$, where for $p=2$, any maximal proper subcomplex of $C_{3}^{0} \vee C_{3}^{0}$ is geometrically realizable in $E^{2}$, as shown in Figure 2.

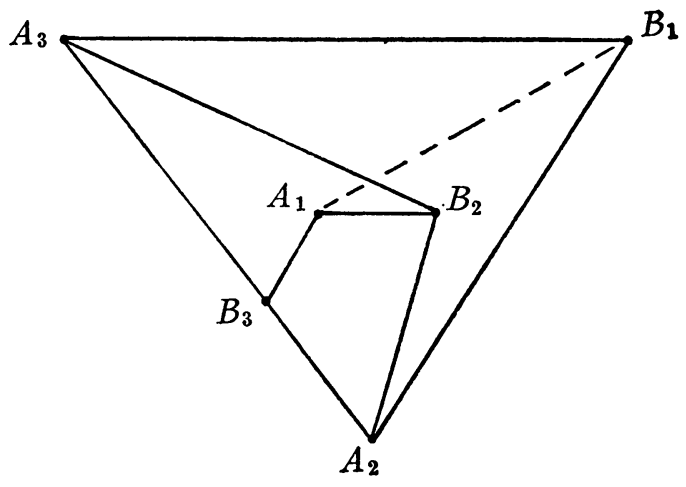

FIGURE 2

For the inductive step we use Theorem 4.

Case II. One of the $n_{i}-s$ is $\neq 1$. Let $n_{1} \neq 1$. The proof is again by induction on $p$, where we begin with $C_{2 n_{1}+1}^{n_{1-1}}$ for which we use Theorem 3 , part (b). For the inductive step we use again Theorem 4.

This completes the proof of Theorem 3.

5. Proof of Theorem 2. The proof is by induction on $p$, for all $n$. Using part (a), Case I of Theorem $3 \mathrm{~b}$, we may assume that not all $n_{i}=1$, and in particular, that $n_{1} \neq 1$. For $p=1, C_{2 n_{1}+1}^{n_{1-1}}$ has the required property for all $n_{1} \neq 1$, by Theorem 3 , part (b).

For the inductive step, we use Theorem 4 or Theorem 5, depending on whether we have to add $C_{3}^{0}$, or $C_{2 n_{j}+1}^{n_{j}-1}$ with $n_{j} \neq 1$.

REMARK To ThEOREM 4. A similar assertion, using $m$ instead of 3 , where $m>3$ is false, since a maximal proper subcomplex of $K \bigvee C_{m}^{0}$ contains a subcomplex isomorphic to $K \bigvee C_{3}^{0}$. But if $K$ is a nice complex, then $K \bigvee C_{3}^{0}$ is a nice complex, hence not even topologically embeddable in $E^{2 n}$.

\section{REFERENCES}

1. A. Flores, Über die Existenz n-dimensionaler Komplexe, die nicht in den $R_{2 n}$ topologisch einbettbar sind, Ergeb. Math. Kolloq. 5 (1932/33), 17-24. 1967.

2. B. Grünbaum, Graphs and complexes, Lectures Notes, Univ. of Washington,

3. E. R. van Kampen, Komplexe in Euklidischen Raumen, Abh. Math. Sem. Univ. Hamburg 9 (1932), 72-78 and 152-153. 
4. C. Kuratowski, Sur le probleme des courbes gauches en topologie, Fund. Math. 15 (1930), 271-283.

5. R. H. Rosen, Decomposing 3-space into circles and points, Proc. Amer. Math. Soc. 11 (1960), 918-928.

6. W. T. Wu, $A$ theory of imbedding immersion and isotopy of polytopes in a Euclidean space, Science Press, Peking, 1965.

UNIVERSITY OF WASHINGTON 